\title{
Theoretical Study of Oxygen Atom Adsorption on a Polycyclic Aromatic Hydrocarbon Using Density-Functional Theory
}

\author{
Mokhammad Fajar Pradipta ${ }^{1,2^{*}}$, Harno Dwi Pranowo ${ }^{1,2}$, Viny Alfiyah ${ }^{1,2}$, and Aulia Sukma Hutama ${ }^{1,2^{* *}}$ \\ ${ }^{1}$ Austrian-Indonesian Centre (AIC) for Computational Chemistry, Faculty of Mathematics and Natural Sciences, \\ Universitas Gadjah Mada, Sekip Utara, Yogyakarta 55281, Indonesia \\ ${ }^{2}$ Department of Chemistry, Faculty of Mathematics and Natural Sciences, Universitas Gadjah Mada, \\ Sekip Utara, Yogyakarta 55281, Indonesia
}

\author{
${ }^{*}$ Corresponding author: \\ email:fajar@ugm.ac.id; \\ aulia.sukma.hutama@ugm.ac.id ${ }^{* *}$ \\ Received: January 20, 2020 \\ Accepted: May 12, 2020
}

DOI: $10.22146 / \mathrm{ijc} .53583$

\begin{abstract}
Potential energy curves (PECs) and energy profiles of atomic $O$ attack on coronene as a model for graphene/graphitic surface and interstellar reaction surface have been computed at the unrestricted $B 3 L Y P / c c-p V D Z$ level of theory to elaborate on atomic $O$ attack mechanism and chemisorption on coronene. The PECs were generated by scanning the $O$ atom distance to the closest carbon atom on "top" and "bridge" positions in the coronene, while fully relaxed geometries in the triplet state were investigated to gain the energy profile. We found that the most favorable geometry as the final product was the chemically bound $O$ on the "bridge" site in the singlet state with an interaction energy of $-29.2 \mathrm{kcal} / \mathrm{mol}$. We recommended a plausible mechanism of atomic $O$ attack and chemisorption reaction on coronene or generally graphitic surface starting from the noninteracting $O$ atom and coronene systems into the chemically bound $O$ atom on coronene.
\end{abstract}

Keywords: graphene oxide; chemisorption; density functional theory

\section{INTRODUCTION}

Single-atom chemical functionalization on graphene [1] has been investigated to alter the graphene properties for many diverse applications [2]. Oxygen atom is one of the most investigated atoms to modify graphene [3] or graphene oxide (GO). It could be applied on electronic devices, for instance, field-effect transistors device [4], thin-film transparent conductor [5], and a light-emitting diode or LED [6]. The GO is feasible to be used as a precursor to synthesize graphene-based functionalized nanosheets via chemical reductions [7]. Thus, an efficient GO synthesis method is a critical issue for electronic materials possessing preferred electric performances. The strong oxidizing agent is used towards graphene [8] or exfoliating the bulk form to layered sheet $[5,7,9]$ in synthesizing GO. The synthesized GO has a diverse distribution of oxygen-contained functional groups, for instance, phenol, carboxylic, lactone, carbonyl, hydroxyl, epoxide, and quinone [3,10-12]. Inhomogeneity of the synthesized GO inhibited uninterrupted electron transportation on the synthesized graphene nanosheets compared to the pristine graphene material, caused by defects in the nanosheets [3]. Direct oxygen plasma exposure to graphene is another synthesis method to obtain GO [1315]. Careful control must be taken when using the plasma oxygenation approach; high-energy species such as $\mathrm{O}^{2+}$ exposure on the graphene could cause irreversible lattice damages and provoke defects on the sheet.

One of the propitious methods to obtain homogeneous graphene oxide is atomic $\mathrm{O}$ exposure to graphene via thermal cracking [16-17]. $\mathrm{O}_{2}$ molecules are splintered into $\mathrm{O}$ atoms by a hot tungsten filament at $\sim 1500{ }^{\circ} \mathrm{C}$ under ultrahigh vacuum conditions. Although it is arduous to obtain the details of structural information and mechanism caused by the extreme experimental condition, a theoretical calculation can provide more insight into the mechanism investigation. Several theoretical investigations of $\mathrm{O}$ insertion on graphene [18-19] and graphite [20-21] with periodic boundary conditions (PBC) have been conducted. Most 
of the reported investigations have focused on the GO nanosheet structures. Still, the atomic oxygen attack reaction mechanism on graphene has not been investigated deeply, which could aid in explicating the GO structure in the experimental results and designing new GO-based materials.

In the field of astrophysics and astrochemistry, polycyclic aromatic hydrocarbons (PAHs) could be used for modeling $2 \mathrm{D}$ reaction surfaces in interstellar space [22-26]. Atomic O plays a significant role in the elementary water formation processes under the interstellar medium [27]. The interaction of PAHs with atomic $\mathrm{O}$ may play a major role in further, a more complicated chemical reaction in the interstellar medium. For example, the elementary process of water formation was proposed due to the adsorption of atomic $\mathrm{O}$ on dust grains [28-29]. Hence, it is interesting to study the interaction between atomic $\mathrm{O}$ and PAHs.

In this theoretical study, we investigated atomic $\mathrm{O}$ attack and chemisorption on coronene $\left(\mathrm{C}_{24} \mathrm{H}_{12}\right)$, a $\mathrm{PAH}$, by using density functional theory (DFT). Coronene has also been consistently employed as a model of graphene for the atom-graphene interaction studies [30-34]. We showed plausible reaction intermediates, transition states, and products. We propose a mechanism of atomic $\mathrm{O}$ attack on graphene in an early stage of graphitic material oxidation to explain the experimentally observed oxygen homogeneity on graphene [16] and probable impacts on astrochemistry.

\section{- COMPUTATIONAL METHODS}

To predict possible stationary points of the $\mathrm{O}$ attack process in the initial stage, we calculated the potential

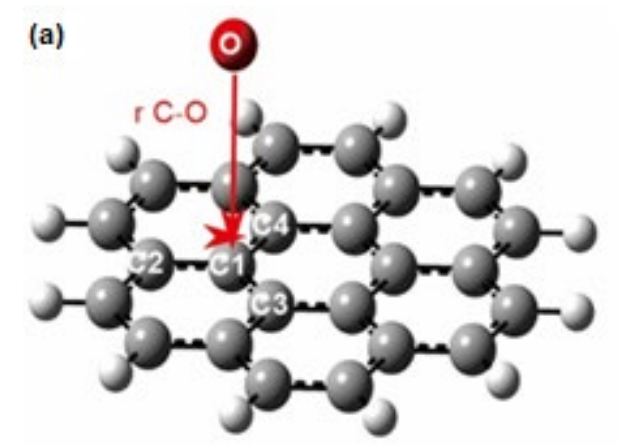

energy scan of atomic $\mathrm{O}$ on coronene $\left(\mathrm{C}_{24} \mathrm{H}_{12}\right)$. There are three possible attack sites on coronene, which are "top", "bridge," and "hollow", as being shown in Fig. 1.

The potential energy calculations on the distance between the $\mathrm{O}$ atom and the coronene sheet at the "top" site or "bridge" site were carried out to observe the interaction of the $\mathrm{O}$ atom that may form a single bond or two single bonds with the $\mathrm{C}$ atom in the coronene sheet respectively, while the attack at "hollow" site was not considered because the $\mathrm{O}$ atom attack at the site will be energetically unfavorable since the interaction energy indicated repulsive as in the case of $\mathrm{H}$ and $\mathrm{F}$ [33-34]. As shown in Fig. 2, the $\mathrm{C}-\mathrm{O}$ distances, $\mathrm{rC}-\mathrm{O}$, are described as the distance between $\mathrm{O}$ and $\mathrm{C} 1$ at the "top" site and between $\mathrm{O}$ and the center of $\mathrm{C} 1$ and $\mathrm{C} 4$ atoms at the "bridge" site, where $\mathrm{rC}-\mathrm{O}=\mathrm{rC} 1-\mathrm{O}=\mathrm{rC} 4-\mathrm{O}$.

$\mathrm{O}$ atom and coronene ground states take triplet state $\mathrm{O}\left({ }^{3} \mathrm{P}\right)$ and closed singlet state, respectively. Both the $\mathrm{O}$ atom and coronene are on the ground state when separated, while the singlet or triplet state is possible when chemical bonds are formed. Therefore, we examined both states to study the reliance of the adsorption reaction mechanism on the contrastive spin states.

Following geometry optimization computation of the coronene sheet, we introduced relaxed scans at "top"

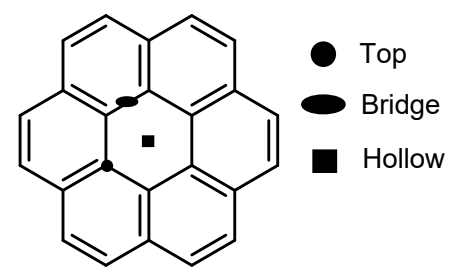

Fig 1. Possible $\mathrm{O}$ attack sites on coronene $\left(\mathrm{C}_{24} \mathrm{H}_{12}\right)$ sheet

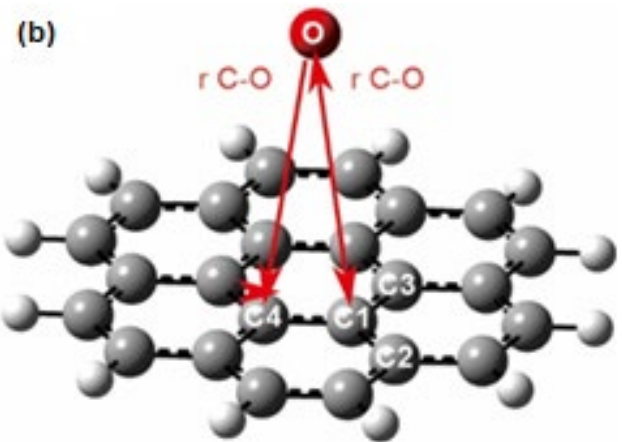

Fig 2. Coordinate definitions of $\mathrm{rC}-\mathrm{O}$ at the (a) "top" and (b) "bridge" sites on coronene 
and "bridge" sites in triplet state with fixing $\mathrm{rC}-\mathrm{O}$ to gain the PECs for the range between the $\mathrm{O}$ and each site. In the case of the singlet state, the $\mathrm{O}$ tended to form bridges with the closest two carbon atoms in the coronene at both attack sites, which prevented from completing relaxed scans at the "top" site correctly. Therefore, we performed single-point calculations based on the geometries obtained by the relaxed scan in the triplet state because geometries in the triplet and singlet state should not be mainly different in general. After the PECs are obtained, the geometries from the local minima or maxima on the PECs in both spin states are optimized and followed by vibrational analyses. In the existing transition states, the intrinsic reaction coordinate (IRC) calculations were conducted to ensure that the generated geometry structures were transition states.

We applied unrestricted B3LYP [35] density functional with the cc-pVDZ basis set to scan the relaxed "top" and "bridge" sites and full optimizing calculations of the stationary points. The B3LYP/cc-pVDZ level of theory is accurate enough to give the adsorption energy because the energies in the analogous system of coronene with $\mathrm{H}$ and $\mathrm{F}$ atom evaluated at the same level of theory, $\mathrm{B} 3 \mathrm{LYP} / \mathrm{cc}-\mathrm{pVDZ}$ were similar to the energies estimated by the correlated $a b$ initio wave function method to the complete basis set limit [33-34]. All computations performed in this work were carried out using Gaussian 09 program package [36]. To compute the bond orders, the wave function (.wfx) file from Gaussian 09 output calculated at B3LYP/cc-pVDZ level of theory was processed using the chargemol code [37].

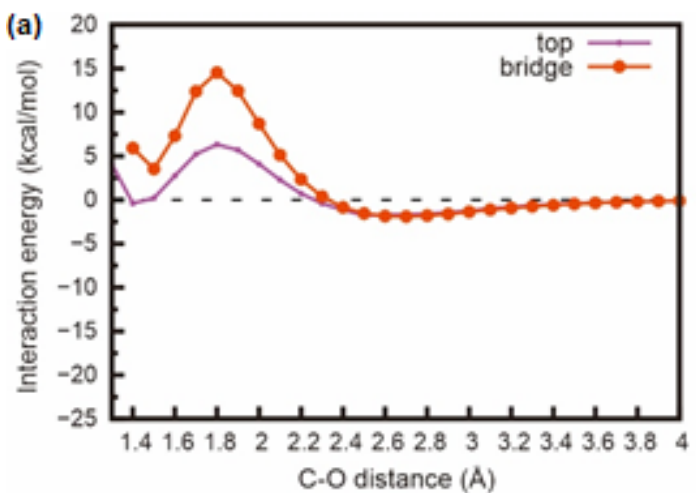

Fig 3. PECs interaction between coronene and $\mathrm{O}$ atom at B3LYP/cc-pVDZ level of theory calculation in (a) triplet and

(b) singlet states

The interaction energies, $E_{\text {int }}$, were evaluated by the following equation:

$\mathrm{E}_{\text {int }}=\mathrm{E}_{\text {coronene- } \mathrm{O}}-\mathrm{E}_{\text {coronene }}-\mathrm{E}_{\mathrm{O}}$

where $\mathrm{E}_{\text {coronene- } \mathrm{O}}, \mathrm{E}_{\text {coronene, }}$ and $\mathrm{E}_{\mathrm{O}}$, are the energies of coronene and $\mathrm{O}$ atom complex, optimized coronene, and $\mathrm{O}\left({ }^{3} \mathrm{P}\right)$, respectively. The bond orders were computed using the density-derived electrostatic and chemical (DDEC6) partitioning method [37-39].

\section{- RESULTS AND DISCUSSION}

\section{Potential Energy Curves from B3LYP/cc-pVDZ Level of Theory}

Based on the geometry, the interaction energy can be considered as van der Waals (vdW) interaction, which is analogous to graphene and $\mathrm{OH}$ radical models [40]. Local maximum energy observed in the PEC at "top" site at $6.3 \mathrm{kcal} / \mathrm{mol}$ and local minimum energy at $0.4 \mathrm{kcal} / \mathrm{mol}$. The $\mathrm{rC}-\mathrm{O}$ distance in local maximum and local minimum energy was detected at $1.8 \AA$ and $1.4 \AA$, respectively. While at the "bridge" site, local maximum energy was observed at $14.5 \mathrm{kcal} / \mathrm{mol}$ with an $\mathrm{rC}-\mathrm{O}$ distance equal to $1.8 \AA$ and local minimum energy of $3.3 \mathrm{kcal} / \mathrm{mol}$ with a shorter $\mathrm{rC}-\mathrm{O}$ distance of $1.5 \AA$ (Fig. 3(a)). Activation barrier of atomic oxygen attack towards coronene and the oxygenated-coronene formation indicated by the maximum and minimum PECs. Furthermore, the PEC scores were collected by single-point computations in the singlet and triplet states at the scanned geometry structures as being shown in Fig. 3(b), a minimum energy of $1.5 \mathrm{kcal} / \mathrm{mol}$ at $\mathrm{rC}-\mathrm{O}=$ $1.4 \AA$ and $-20.4 \mathrm{kcal} / \mathrm{mol}$ at $\mathrm{rC}-\mathrm{O}=1.5 \AA$ were obtained

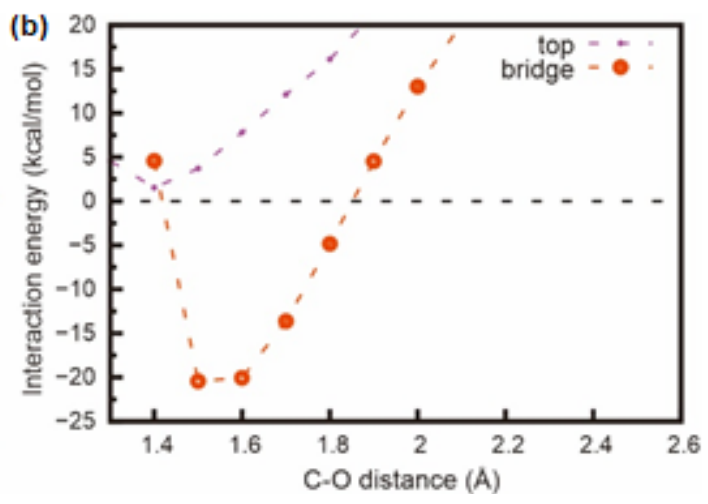

Mokhammad Fajar Pradipta et al. 
for "top" site and "bridge" site, respectively, signifying the formation of coronene-O adduct in the system.

The interaction energy of the singlet state goes up with increasing $\mathrm{C}-\mathrm{O}$ distance because at far coronene- $\mathrm{O}$ distance, the system converges to these states:

coronene $\left({ }^{1} A^{\prime}\right)+O\left({ }^{1} D\right)$

while in the triplet state, the system converges to

coronene $\left({ }^{1} \mathrm{~A}^{\prime}\right)+\mathrm{O}\left({ }^{3} \mathrm{P}\right)$

It is evident that singlet $\mathrm{O}\left({ }^{1} \mathrm{D}\right)$ is higher in energy than triplet $\mathrm{O}\left({ }^{3} \mathrm{P}\right)$. Unfortunately, for the singlet state PECs, we cannot show the energy after $2.6 \AA$ since the spin squared expectation values $\left(\mathrm{S}^{2}\right)$ exceed 0.1 at far $\mathrm{C}-\mathrm{O}$ distance rendering the single determinant wave function unreliable. Such a problem can be resolved by employing a multireference wave function level of theoretical calculations. Unfortunately, the calculations require high computational costs, and our current infrastructure cannot support the calculations. Theoretically, the energy should converge to approximately $45 \mathrm{kcal} / \mathrm{mol}$, which is the $\mathrm{O}\left({ }^{3} \mathrm{P}\right) \rightarrow \mathrm{O}\left({ }^{1} \mathrm{D}\right)$ transition energy.

On the other hand, the values of the triplet state are between 2.00 and 2.005 for all points in the triplet PECs, implying that the triplet state has small properties of multiconfiguration. Hence, we could employ the single determinant calculation for all points in the triplet state. In contrast, the single determinant (DFT) approach can only be used for the coronene-O binding region of the singlet state.

It is known that the $\mathrm{H}$ atoms significantly modify electronic properties, e.g., coronene has a substantial quadrupole moment and significant HOMO-LUMO gap while graphene is a zero-gap semiconductor. However, our focus is on the potential energy curve (PEC) for the approach of an $\mathrm{O}$ atom towards coronene as a graphene model to provide accurate reference values. We confirmed that using a larger model, i.e., circumcoronene $\left(\mathrm{C}_{54} \mathrm{H}_{18}\right)$, the profile of $\mathrm{PEC}$ for $\mathrm{O}$ atom chemisorption on circumcoronene was similar to that of coronene. Termination of the coronene model by $\mathrm{H}$ atoms is unavoidable for practical reasons. Moreover, nonhydrogen terminated finite molecular graphene models have significantly different orbital types compared to infinite graphene [41].
The stationary points aforementioned are not the true minimum energy because they were obtained with the distance of the $\mathrm{C}-\mathrm{O}$ bond fixed. To discuss details of the $\mathrm{O}$ attack process, we used the obtained maximum and minimum structures as initial geometries for further full optimizing calculations described below.

\section{Optimized structures and energy diagram}

Based on the PECs data, we could conjecture diagram energy for atomic $\mathrm{O}$-coronene interaction as a graphene model for all sites. From the PECs at the 'top' site, we obtained the stationary points, and they are described as follows $\mathrm{rC}-\mathrm{O}=1.4 \AA$ as oxidized coronene, $\mathrm{rC}-\mathrm{O}=2.5 \AA$ as vdW complex, and the in-between medium distance to form $\mathrm{C}-\mathrm{O}$ bond, respectively. For the "bridge" site, the points could be around $\mathrm{rC}-\mathrm{O}=1.5 \AA$ as oxidized coronene, $\mathrm{rC}-\mathrm{O}=2.6 \AA$ as vdW complex, and the in-between medium range to form $\mathrm{C}-\mathrm{O}$ bond and alter the spin state. Optimized stable species are being labeled in alphabetical order. The structural geometries at transition states (TS) are represented following the reactant and product's name, respectively. The energy diagram was constructed by utilizing the relative energies to the sum of the total energy of coronene in singlet state and $\mathrm{O}\left({ }^{3} \mathrm{P}\right)$ states.

The triplet states in Fig. 4(a) visualized that the van der Waals complexes at the "top" and the "bridge" sites approached the same species of A. In the "top" site, we observed the $\mathrm{O}$ atom with the $\mathrm{C} 1-\mathrm{O}$ distance of $2.67 \AA$ with a value of $-2.0 \mathrm{kcal} / \mathrm{mol}$ for their interaction energy. The structure geometry at the local maximum (about $\mathrm{rC}-\mathrm{O}=1.80 \AA$ ) on the PEC of the "top" site being optimized and the first-order transition state labeled as TS(AB) observed, and the $\mathrm{O}$ atom is located in the "top" site with the distance of $\mathrm{rC} 1-\mathrm{O}$ is $1.82 \AA$ and $6.1 \mathrm{kcal} / \mathrm{mol}$ for its interaction energy. However, the optimized geometry structure observed converging into an unexpected second-order transition state for the case of the "bridge" site. Therefore, this peculiar structure will not be discussed in the remaining discourse. Two stable geometries of the coronene-O adduct were obtained and labeled as B and C from the initial geometry structures of the oxidized coronene with the distance around $\mathrm{rC}$ $\mathrm{O}=1.4 \AA$ at the "top" and "bridge" sites. The $\mathrm{B}$ and $\mathrm{C}$ were 
(a)

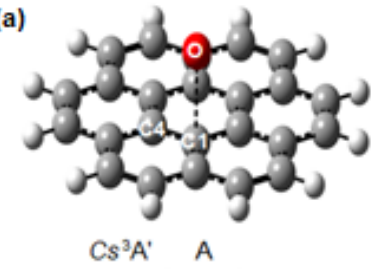

r C1.0: $2.67 \mathrm{~A}$
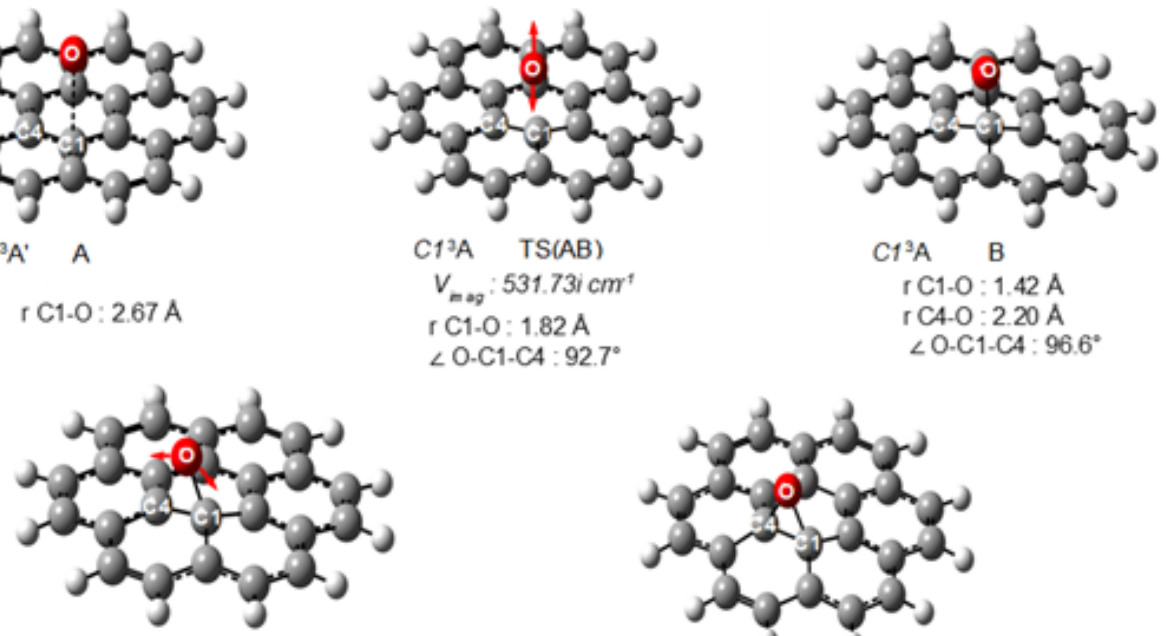

$\mathrm{C}^{3} \mathrm{~A} \quad \mathrm{TS}(\mathrm{BC})$

$V_{m \infty x}: 522.58 i \mathrm{~cm}^{\dagger}$

r C1-O: $1.43 \mathrm{~A}$

I C4-O: $1.72 \mathrm{~A}$

$\angle \mathrm{O}-\mathrm{C} 1-\mathrm{CA}: 723^{\circ}$

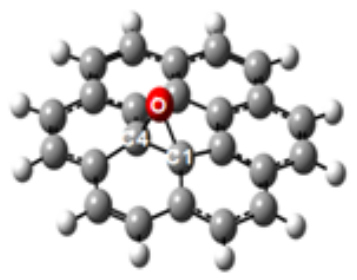

$\mathrm{C}^{3} \mathrm{~A} \quad$ (C)

I C1-O : $1.48 \mathrm{~A}$

r C4-O: $1.48 \mathrm{~A}$

$\angle \mathrm{O}-\mathrm{C} 1-\mathrm{C} 4: 59.7^{\circ}$

(b)


r C1-O: 1.43 A

r C2-O: $1.44 \mathrm{~A}$

$\angle \mathrm{O}-\mathrm{C} 1-\mathrm{C} 2: 57.4^{\circ}$

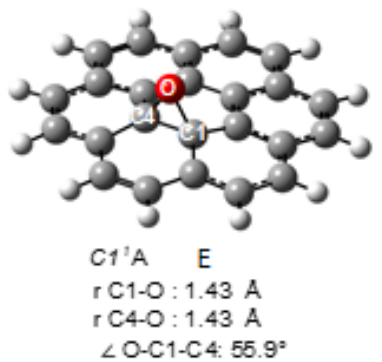

C1'A TS(DE)

$V_{\text {mag }}: 695.25 \mathrm{icm}^{-1}$

r C1-0: $1.42 \mathrm{~A}$

r C2-O: $224 \mathrm{~A}$

r C4O: $216 \mathrm{~A}$

$\angle \mathrm{OC} 1-\mathrm{C} 4: 95.7^{\circ}$

$\angle \mathrm{O}-\mathrm{C} 1-\mathrm{C} 2: 100.3^{\circ}$

Fig 4. Optimized intermediates and transition states with their point group, state, and specified geometrical structures in (a) triplet and (b) singlet states. Red-colored arrows were being drawn to represent the constructed frequencies modes for the transition states (TS).

observed to have $-1.8 \mathrm{kcal} / \mathrm{mol}$ and $3.3 \mathrm{kcal} / \mathrm{mol}$ interaction energy, respectively. Furthermore, a firstorder transition state structure between $\mathrm{B}$ and $\mathrm{C}$ was obtained and labeled as TS(BC) with the distance of $\mathrm{rC1}$ $\mathrm{O}=1.43 \AA$ and $\mathrm{rC} 4-\mathrm{O}=1.73 \AA$ and $6.1 \mathrm{kcal} / \mathrm{mol}$ as its interaction energy. This result was unexpected we obtained from the relaxed scan PECs. As for the singlet state in Fig. 4(b), two possibilities of structures were available at the "bridge" site, which were labeled as the "edge-bridge" (D) and the "graphitic-bridge" site (E). The "edge-bridge" structure (D) were calculated by optimizing the structure of $\mathrm{rC}-\mathrm{O}=1.40 \AA$ on the PEC of the "top" site since it is observed as an unstable species in the singlet state. We also optimizing the geometry 
structure of $\mathrm{rC}-\mathrm{O}=1.50 \AA$ on the PEC of the "bridge" site to obtain the "graphitic-bridge" structure. Both the $\mathrm{D}$ and E geometries were recognized to exhibit epoxide structures with the $-35.0 \mathrm{kcal} / \mathrm{mol}$ and $-29.2 \mathrm{kcal} / \mathrm{mol}$ for the interaction energy values, respectively. The geometry of the transition state (TS) between the D and E structures, labeled as TS(DE), was optimized with the distance, $\mathrm{rCl}-\mathrm{O}=1.42 \AA$, with an interaction energy value of $1.2 \mathrm{kcal} / \mathrm{mol}$. The complete energy surface of the stationary points is shown in Fig. 5.

Other theoretical studies report that the interaction energy of the graphene model introduced with a single $\mathrm{O}$ atom is ca. $-55 \mathrm{kcal} / \mathrm{mol}$ [42]. The discrepancy with our computed value might be caused by the differences in the computational methods. For a test, we compared the interaction energy between coronene and $\mathrm{O}$ atom using the plane wave PAW method [43] using PBE functional [44] (PBE-PAW) and PBE/cc-pVDZ methods. It turns out that the former gives the interaction energy of
$-54.1 \mathrm{kcal} / \mathrm{mol}$ while the latter gives $-48.0 \mathrm{kcal} / \mathrm{mol}$. Thus, we might suspect that the previous reports were using PBE-PAW as their level of theory. PBE is also known to underestimate the interaction energy, as we encountered earlier [33-34].

\section{The Possible Intersystem Crossing Pathway (ISCP)}

It is conclusive that the coronene-O system is stable on its triplet state at far $\mathrm{C}-\mathrm{O}$ distance, while the coronene-O binding region is stable in the singlet state. Therefore, it is reasonable to address a possible ISC mechanism during the reaction. Although the multireference (MR) calculations to describe the energetics are desirable, the high computational cost of such methods inhibits us from conducting the calculations for the current systems. In any case, the DFT level of theory seems to be good enough to predict qualitatively and even quantitatively the correct energy ordering and potential energy surfaces (PES) of species

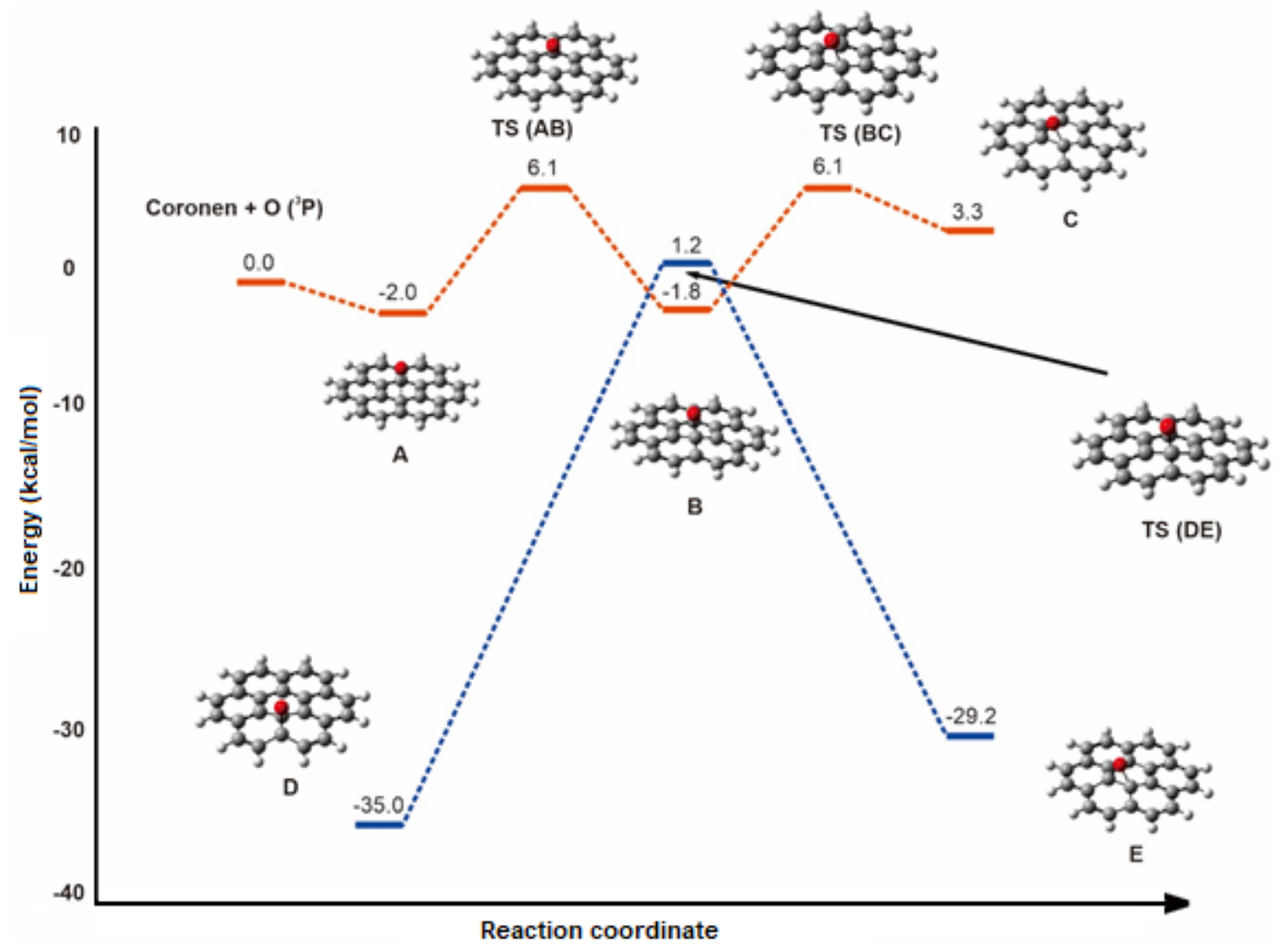

Fig 5. Adduction of $\mathrm{O}$ atom on coronene structure in the triplet (red) and singlet (blue) states potential energy diagram $(\mathrm{kcal} / \mathrm{mol})$ 
with dissimilar spin states in comparison with more expensive high-level MR wave function theories such as RASPT2 and CASPT2 [45-48].

To figure out the possible location of the ISC point, the PECs from the relaxed scan and the interaction energy at the stationary points were compared. Fig. 6 shows the comparison between the triplet and singlet states at each adsorption site. As shown in Fig. 6(a), there is an additional point at ca. $1.43 \AA$ with $-29.2 \mathrm{kcal} / \mathrm{mol}$ at the singlet PEC corresponds to the interaction energy at $\mathrm{E}$ geometry. The interaction energy at $\mathrm{C}$ geometry nearly overlaps the interaction energy at $\mathrm{rC}-\mathrm{O}=1.40 \AA$. The figure shows that for the bridge site, the PECs of the triplet and singlet states cross at approximately $\mathrm{C}-\mathrm{O}$ distance of 1.95 and $1.4 \AA$ with the interaction energy of ca. 13 and $5 \mathrm{kcal} / \mathrm{mol}$, respectively. For this site, the first possible ISC occurs before the direct insertion of the $\mathrm{O}$ atom from the vdW complex to the triplet bridge site. Second possible ISC point for the bridge site is at $\mathrm{rC}-\mathrm{O}=1.4 \AA$ with the interaction energy ca. $5 \mathrm{kcal} / \mathrm{mol}$. However, if the ISC occurs via the second ISC point, the system must overcome the higher energy barrier. Thus, if the ISC occurs via direct bridge site insertion, it should occur via the first ISC point. This ISC point is a spin-change barrier type [46].

Fig. 6(a) shows the comparison between singlettriplet states at the top adsorption site. Interestingly, the singlet and triplet PECs do not cross along the top site pathway, which could mean that the ISC should occur starting from the triplet minimum for the top site. However, since the energy barrier of the $\mathrm{O}$ insertion from $\mathrm{vdW}$ complex to the top site, i.e., interaction energy at

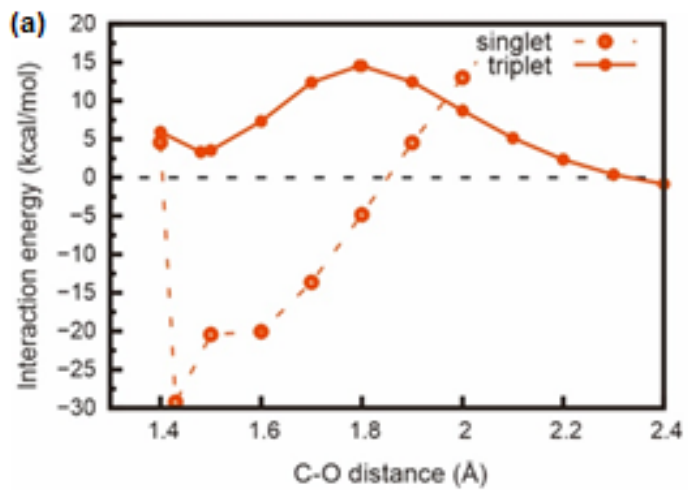

Fig 6. PECs interaction graphs between coronene and oxygen atom at B3LYP/cc-pVDZ level of theory at the (a) bridge and (b) top sites

Mokhammad Fajar Pradipta et al.

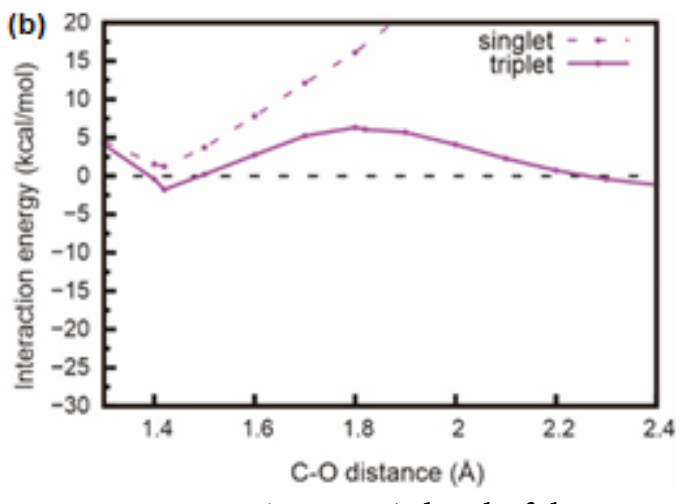

$\mathrm{TS}(\mathrm{AB})$ geometry is lower than the spin-change barrier of the bridge site by ca. $7 \mathrm{kcal} / \mathrm{mol}$, it is most likely that the ISC occurs via the adsorption from the top site.

To further evaluate the ISC process from the top adsorption site, the PECs were computed in the singlet and triplet states by scanning the energy along with the triplet top site minimum geometry (B) to a singlet geometry. From Fig. 4, it is evident that the B and $\mathrm{TS}(\mathrm{DE})$ geometries are nearly identical. Therefore, we consider that the ISC occurs along $B \rightarrow T S(D E)$ pathway. We consider two $\mathrm{B} \rightarrow \mathrm{TS}(\mathrm{DE})$ pathways. The first pathway is the direct $B \rightarrow T S(D E)$ pathway. This pathway is mainly controlled by the $\mathrm{O}-\mathrm{C} 1-\mathrm{C} 4$ and $\mathrm{O}-\mathrm{C} 1-\mathrm{C} 2$ bond angles changes since the $\mathrm{O}-\mathrm{C} 1$ bond length and other important internal coordinates are nearly identical. The second pathway is the $\mathrm{B} \rightarrow \mathrm{TS}(\mathrm{DE})$ pathway via $\mathrm{F}$ geometry (Fig. 7), dubbed as $\mathrm{B} \rightarrow \mathrm{F} \rightarrow$ TS(DE) pathway. F geometry is the output geometry from the relaxed scan at fixed $\mathrm{rC}-\mathrm{O}=1.3 \AA$ in the triplet state. We consider this geometry since the energy in the singlet and triplet states at F geometry is nearly identical from the PEC. Also, if the incoming atom has enough kinetic energy, the system can undergo the reflection and back chemisorption process as in the case of adsorption of atomic $\mathrm{H}$ on graphene [49], which occurs at a shorter $\mathrm{C}-\mathrm{H}$ distance than the equilibrium distance. Therefore, in our case, the ISC may also occur at a shorter $\mathrm{C}-\mathrm{O}$ bond distance. The second pathway is mainly controlled by $\mathrm{C}-\mathrm{O}$ bond shortening and elongation. The pathways were prepared by the following internal coordinate interpolation: 


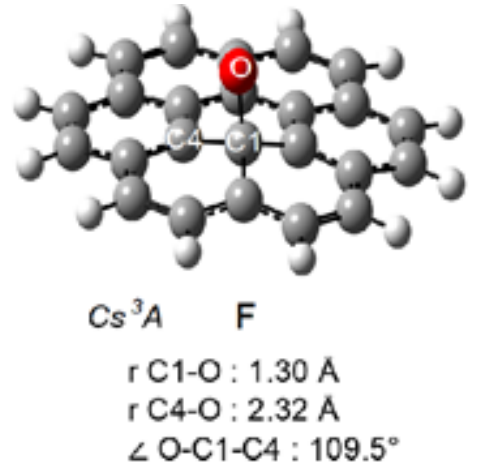

Fig 7. The F geometry

$\mathrm{C}=x \mathrm{C}(\mathrm{E})+(1-x) \mathrm{C}(\mathrm{TS}(\mathrm{DE}))$

where $\mathrm{C}$ is the internal coordinates between $\mathrm{E}$ and $\mathrm{TS}(\mathrm{DE}), \mathrm{C}(\mathrm{E})$ and $\mathrm{C}(\mathrm{TS}(\mathrm{DE}))$ are the optimized internal coordinates of $\mathrm{E}$ and $\mathrm{TS}(\mathrm{DE})$, respectively, and $x$ is the weight coefficient $(0 \leq x \leq 1)$.

Figure 8 shows a reaction pathway from triplet top site adsorption minimum. Fig. 8(a) visualized that the energy of the triplet state increases monotonically until the TS(DE) geometry while the energy of the singlet state decreases. The crossing occurs at proximity of TS(DE) geometry with the barrier energy ca. $3.0 \mathrm{kcal} / \mathrm{mol}$ relative to $\mathrm{B}$ triplet state energy. Fig. 8(b) reveals that at $\mathrm{F}$ geometry, the energy difference is only $0.5 \mathrm{kcal} / \mathrm{mol}$. However, the energy does not seem to cross until the proximity to TS(DE) geometry. Given that the error bar of the B3LYP method is $1-3 \mathrm{kcal} / \mathrm{mol}$, it is reasonable to consider a pathway through a bond shortening mechanism since the energy may cross along the path. Another consideration is that the crossing occurs at an even shorter $\mathrm{C}-\mathrm{O}$ distance. In any case, the crossing via bond shortening mechanism must require higher energy to overcome the barrier than the direct mechanism.

Recent theoretical studies of $\mathrm{O}\left({ }^{3} \mathrm{P}\right)+\mathrm{C}_{2} \mathrm{H}_{4}$ reactions suggested that the ISC could occur at the crossing points where it is observed that the spin state of acetyl biradical $\bullet \mathrm{CH}_{2} \mathrm{CH}_{2} \mathrm{O}$. species transformed from initial triplet minimum state towards an open-shell singlet saddle point [50-52]. Comparable spin state transformation in the $\mathrm{O}$ atom attack with graphite cluster was proposed by Ehrenfest dynamics simulation via time-dependent DFT (TD-DFT) computation, and the range between $\mathrm{O}$ atom with the graphite model surface was observed at ca. $1.2 \AA$ [21] suggests an ISC pathway via bond shortening mechanism since the bond is shorter than the equilibrium $\mathrm{C}-\mathrm{O}$ bond length for a high-energy oxygen collision reaction.

\section{Electronic Structure Changes during Reaction}

To elucidate the electronic structure changes during the reaction, we conducted natural bond orbital (NBO) [53] and bond order analyses for the coronene$\mathrm{O}$ system along the proposed reaction pathway. Herein, we focused on the NBOs and bond orders of $\mathrm{C} 1$ and its neighboring carbon atoms $(\mathrm{C} 2, \mathrm{C} 3$, and $\mathrm{C} 4)$ with the incoming $\mathrm{O}$ atom.

The NBOs results of $\mathrm{C} 1$ to its adjacent carbon bonds in the geometry optimized, isolated coronene structures are expressed as follows

$$
\begin{aligned}
& \sigma_{\mathrm{C}_{1}=\mathrm{C}_{2}}=0.7071\left(\mathrm{sp}^{2.01}\right) \mathrm{C} 1+0.7071\left(\mathrm{sp}^{2.01}\right) \mathrm{C} 2 \\
& \sigma_{\mathrm{C}_{1}=\mathrm{C}_{3}}=0.7071\left(\mathrm{sp}^{2.01}\right) \mathrm{C} 1+0.7071\left(\mathrm{sp}^{2.01}\right) \mathrm{C} 3 \\
& \sigma_{\mathrm{C}_{1}=\mathrm{C}_{4}}=0.7108\left(\mathrm{sp}^{2.01}\right) \mathrm{C} 1+0.7034\left(\mathrm{sp}^{2.01}\right) \mathrm{C} 4
\end{aligned}
$$

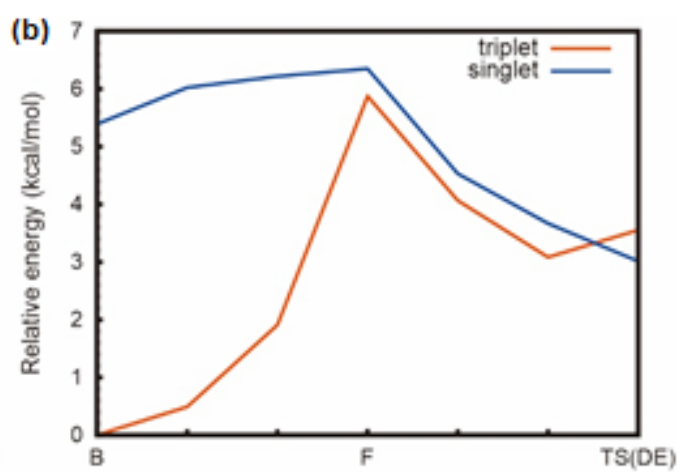

Fig 8. The PECs of possible ISC pathway from triplet top site adsorption minimum (A) to singlet top site geometry (TS(DE)) via (a) direct top and (b) bond shortening mechanism. The energy of B geometry is taken as the reference 
shown that the bonds exhibit strong characteristics of $\mathrm{sp}^{2}$ orbital. Upon reaching the top triplet minimum (B), The NBOs results of $\mathrm{C} 1$ to its adjacent carbon bonds changes to

$$
\begin{aligned}
& \sigma_{\mathrm{C}_{1}-\mathrm{C}_{2}}=0.7081\left(\mathrm{sp}^{2.53}\right) \mathrm{C} 1+0.7062\left(\mathrm{sp}^{2.32}\right) \mathrm{C} 2 \\
& \sigma_{\mathrm{C}_{1}-\mathrm{C}_{3}}=0.7064\left(\mathrm{sp}^{2.46}\right) \mathrm{C} 1+0.7078\left(\mathrm{sp}^{2.26}\right) \mathrm{C} 3 \\
& \sigma_{\mathrm{C}_{1}-\mathrm{C}_{4}}=0.7006\left(\mathrm{sp}^{2.85}\right) \mathrm{C} 1+0.7136\left(\mathrm{sp}^{2.29}\right) \mathrm{C} 4
\end{aligned}
$$

The NBOs indicate that there is observed a shift from $\mathrm{sp}^{2}$ to $\mathrm{sp}^{3}$, but the shift transition is not complete. The NBOs of the final product (E) are

$\sigma_{\mathrm{C}_{1}-\mathrm{C}_{2}}=0.7122\left(\mathrm{sp}^{1.94}\right) \mathrm{C} 1+0.7020\left(\mathrm{sp}^{2.28}\right) \mathrm{C} 2$

$\sigma_{\mathrm{C}_{1}-\mathrm{C}_{3}}=0.7080\left(\mathrm{sp}^{1.96}\right) \mathrm{C} 1+0.7062\left(\mathrm{sp}^{2.21}\right) \mathrm{C} 3$

$\sigma_{\mathrm{C}_{1}-\mathrm{C}_{4}}=0.7078\left(\mathrm{sp}^{6.65}\right) \mathrm{C} 1+0.7064\left(\mathrm{sp}^{6.34}\right) \mathrm{C} 4$

Interestingly, at the bridge site minimum, the bonds hybridization return to the $\mathrm{sp}^{2}$. The drastic change comes from the $\sigma_{\mathrm{Cl}-\mathrm{C} 4}$ bond. The $\mathrm{NBO}$ of this bond changes from $\mathrm{sp}^{3}$ type hybrid to more dominated by $\mathrm{p}$ orbital, which means that the $\mathrm{C} 1-\mathrm{C} 4$ bond in this structure is weaker due to weaker s character.

The bond orders of the selected bonds are listed in Table 1. The presented results can complement the NBO analyses. In the free-standing pristine coronene, the aromatic $\mathrm{C}-\mathrm{C}$ bond orders are uniform with the bond order value of 1.1864. Upon insertion of the $\mathrm{O}$ atom to form the vdW complex, the $\mathrm{C}-\mathrm{C}$ bond order values are slightly reduced to $1.14-1.17$. Additionally, there is no $\mathrm{C}-\mathrm{O}$ bond formation since the $\mathrm{C}-\mathrm{O}$ bond order is below 0.1 . At the $\mathrm{B}$ structure. The most significant change is the weakening of the $\mathrm{C}-\mathrm{C}$ bond around the adsorption site also the formation of the $\mathrm{C} 1-\mathrm{O}$ bond, and the formation of a weak $\mathrm{C} 4-\mathrm{O}$ bond. The aromatic bonds change to the single bond character as shown by the bond order values $(0.85-1.03)$. The bond order value of the $\mathrm{C}-\mathrm{O}$ bond confirms that the $\mathrm{C} 1-\mathrm{O}$ is a single bond.
At the final product geometry $\mathrm{E}$, the $\mathrm{C} 1-\mathrm{C} 2$ and C1-C3 bonds become closer to aromatic bonds. The results are consistent with the NBO results as presented by the hybridization. On the other hand, the adsorbed $\mathrm{O}$ atom on coronene requires both $\mathrm{C} 1$ and $\mathrm{C} 4$ atoms to share the electron, causing the bond weakening of the $\mathrm{C} 1-\mathrm{C} 4$ bond. In this structure, the $\mathrm{C}-\mathrm{O}$ individual bond is imperceptibly weaker than the single $\mathrm{C} 1-\mathrm{O}$ bond in the $\mathrm{B}$ geometry. However, since there are two $\mathrm{C}-\mathrm{O}$ bonds in the E geometry, the total $\mathrm{C}-\mathrm{O}$ bond is stronger.

To confirm the spin location during the reaction, the spin density distribution $(\Delta \rho)$ is analyzed. The spin density is defined as a subtraction operation of the density of $\alpha$ and $\beta$ spin:

$\Delta \rho=\rho_{\alpha}-\rho_{\beta}$

The spin density of vdW complex A shows that spin is localized at the $\mathrm{O}$ atom (Fig. 9). The spin density of $\mathrm{O}$ at the vdW complex is 1.9 , indicating that most spin is located on the $\mathrm{O}$ atom. At the triplet top site $\mathrm{B}$ geometry, the spin density of the $\mathrm{O}$ atom decreases to 0.92 . This value of the spin density suggested that there is an unpaired electron in the $\mathrm{O}$ atom while the other

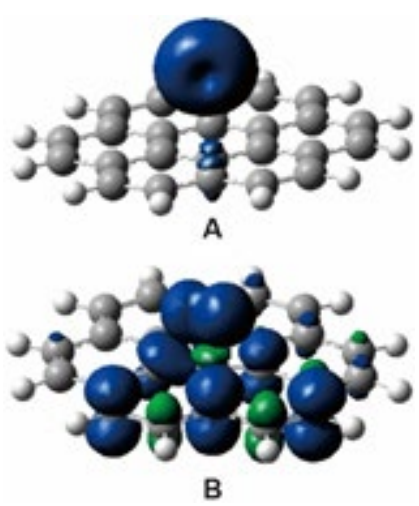

Fig 9. Spin density distribution of the van der Waals complex A and triplet top site minimum B geometries

Table 1. Bond order values of the selected bonds of the minima structures

\begin{tabular}{lccccc}
\hline \multirow{2}{*}{ Structure } & \multicolumn{5}{c}{ Bond orders } \\
\cline { 2 - 6 } & $\mathrm{C} 1-\mathrm{C} 2$ & $\mathrm{C} 1-\mathrm{C} 3$ & $\mathrm{C} 1-\mathrm{C} 4$ & $\mathrm{C} 1-\mathrm{O}$ & $\mathrm{C} 4-\mathrm{O}$ \\
\hline Coronene & 1.1864 & 1.1864 & 1.1864 & - & - \\
$\mathrm{A}$ & 1.1433 & 1.1714 & 1.1714 & 0.0624 & 0.0162 \\
$\mathrm{~B}$ & 0.8716 & 0.9037 & 0.8499 & 1.0043 & 0.1173 \\
$\mathrm{E}$ & 1.0269 & 1.0432 & 0.5686 & 0.8954 & 0.8954 \\
\hline
\end{tabular}


unpaired electron is delocalized onto the coronene (Fig. 9). In the final product, the $\alpha$ and $\beta$ spin are distributed equally to the system, which means the spin density is zero for the entire system. This confirms the non-magneticity of the final product as observed in the experiment.

\section{Impact on Graphene Oxidation Mechanism}

Furthermore, we direct our attention to the atomic $\mathrm{O}$ attack with low energy in real experiments, whereupon the $\mathrm{O}$ atom would not generate defects on the coronene or any other graphitic surfaces. Based on the potential surface in Fig. 4, we could expect oxygen chemosorption on graphene due to the atomic $\mathrm{O}$ atom direct attack on the sheet. Oxygen atom on its ${ }^{3} \mathrm{P}$ state approaching graphene to develop such a precursor complex (A) at the range of $\mathrm{rC} 1-\mathrm{O}=2.67 \AA$. After passing the activation barrier point, at $6.1 \mathrm{kcal} / \mathrm{mol}$ in the distance of $\mathrm{rC} 1-\mathrm{O}=$ $1.82 \AA$, the range between the $\mathrm{O}$ atom and the coronene is reduced to $\mathrm{rC} 1-\mathrm{O}=1.42 \AA$, and a frail coronene-O complex formed and labeled as $\mathrm{B}$. The $\mathrm{B}$ structure is a biradical complex with a triplet state and $-1.8 \mathrm{kcal} / \mathrm{mol}$ for its interaction energy. On the B structure, an unpaired electron is observed to localized on the $\mathrm{O}$, and the other radical is delocalized around the coronene. Following the B structure formation, there is a probability of developing an epoxide structure.

The first plausible reaction mechanism is a ringclosure reaction between $\mathrm{C} 4$ and the $\mathrm{O}$ atom to form a $\mathrm{C} 4-\mathrm{O}$ bond, and the reaction results in the epoxide-like forms complex $\mathrm{C}$ on its triplet state. The second plausible pathway is an intersystem crossing (ISC) that occurred from the triplet state to the singlet state due to the energy difference between the $\mathrm{B}$ structure and the TS(DE) geometry only several $\mathrm{kcal} / \mathrm{mol}$, and the $\mathrm{B}$ structure are alike to the TS(DE) geometry. The ISC likely occurs via the direct mechanism.

After the ISC, the D or E structure could be formed by a plausible ring-closure reaction. Either $\mathrm{D}$ or $\mathrm{E}$ structure should form as a major product in the singlet state due to epoxide formation in the singlet state, D or E, which is considerably more stable than on its triplet state, C. However, we do not have to consider the difference between $\mathrm{D}$ and $\mathrm{E}$ because they are the same structures on graphene. The results generated by our investigations correspond with the precursory work, which stated that the ground state of graphene oxide (GO) structure has a nonmagnetic property [13].

According to our work, we assume that the atomic $\mathrm{O}$ attack on graphene generates graphene oxide (GO) with epoxy structure as the major product since the formation of poorly $\mathrm{O}$ atom adsorption on the "top" site of graphene in a triplet state could quickly encounter relocation to the "bridge" in singlet state due to the notable energy difference among those states whereas the latter is substantially lower. While comparison with the experimental barrier is desirable, to the best of our awareness, there is no systematic investigation on the kinetic of $\mathrm{O}$ adsorption on graphene or PAHs. Therefore, the energy barrier may be estimated by the reaction condition. According to the kinetic theory of gas, the kinetic energy expression of monoatomic gas, $\mathrm{E}_{\mathrm{k}}$ $=3 / 2 \mathrm{k}_{\mathrm{b}} \mathrm{T}\left(\mathrm{k}_{\mathrm{b}}\right.$, Boltzmann constant $)$ with the experimental reaction condition of ca. $1500{ }^{\circ} \mathrm{C}$ [16], corresponds to the kinetic energy of ca. $5.3 \mathrm{kcal} / \mathrm{mol}$, which is necessary to pass the computed barrier, $6.1 \mathrm{kcal} / \mathrm{mol}$.

\section{Impact on Astrochemistry}

Oxygen atom interaction with the PAH surface can be illustrated in Fig. 10. The $\mathrm{O}$ atom originally from the interstellar cloud is obliged to the surface of PAH via vdW interaction, and the $\mathrm{O}$ atom is located at 2.5-2.6 $\mathrm{A}$ above the surface. If the $\mathrm{O}$ atom receives kinetic energy larger than $6 \mathrm{kcal} / \mathrm{mol}$ to conquer the first barrier and the spinchange barrier, the atom can be covalently bound on the surface, and as a result, the $\mathrm{C}-\mathrm{O}$ bond is formed. In this case, both direct and bond shortening ISC can occur depending on the kinetic energy of the incoming $\mathrm{O}$ atom.

The first $\mathrm{O}$ insertion barrier energy value is similar to the $\mathrm{H}$ atom insertion barrier energy value to PAHs as reported by Tachikawa and Wang et al. [30,34] with 5-7 kcal/mol while $\mathrm{O}$ atom binds stronger than $\mathrm{H}$ atom. Since in case of water formation in interstellar medium requires both adsorptions of $\mathrm{O}$ and $\mathrm{H}$ atoms on the substrate [28-29], the adsorption of oxygen and hydrogen atoms on PAHs might be an alternative route of water formation in the case of the interstellar medium. 


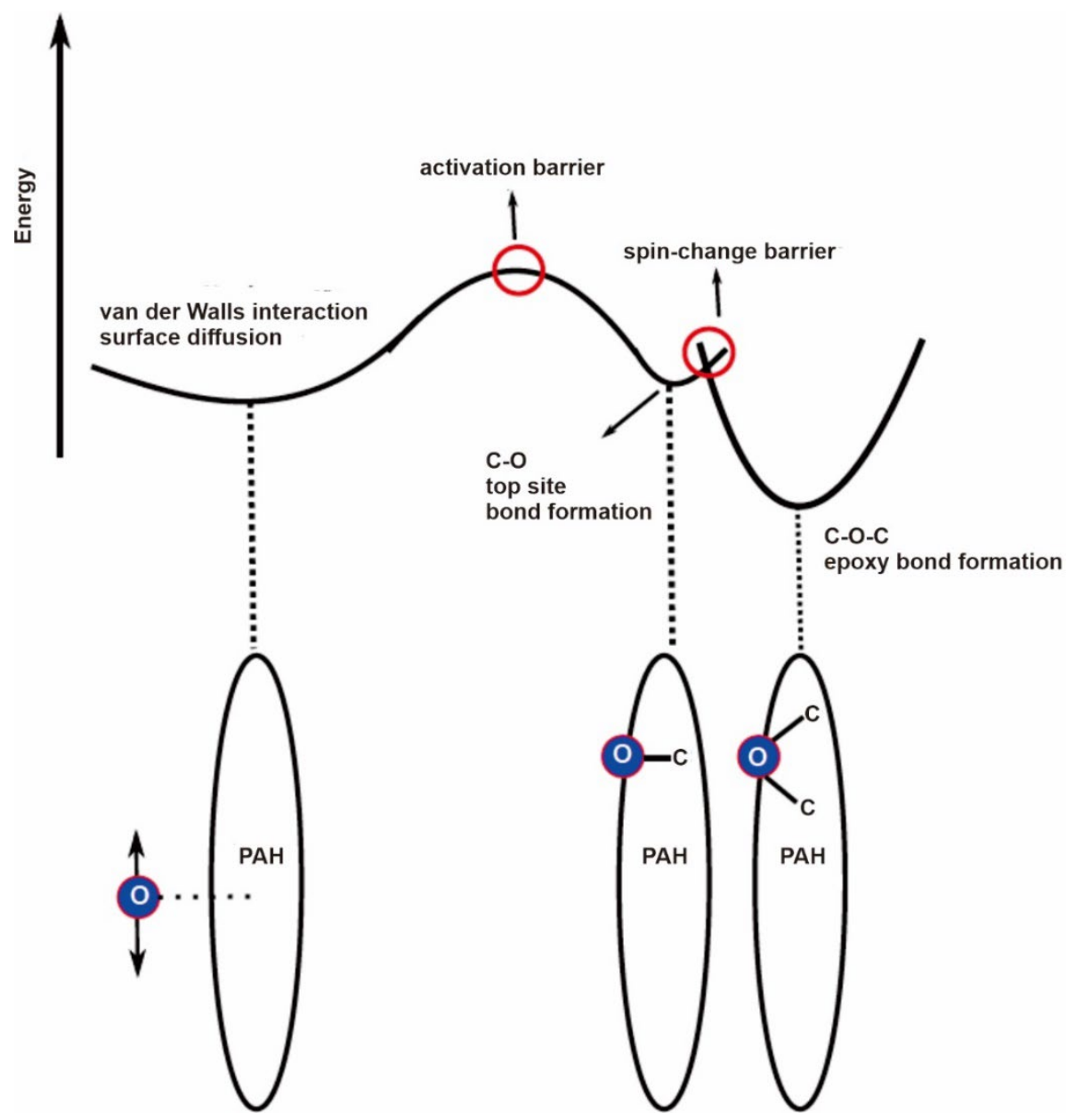

Fig 10. Depiction of the interaction of $\mathrm{O}$ atom with PAHs

\section{CONCLUSION}

We theoretically suggested a plausible atomic oxygen attack and chemisorption mechanism on the coronene-based structure on the PECs computed by DFT at the "top" and "bridge" sites on the coronene structure. We ensured that the coronene-O system has a stable spin state on its triplet state at the far $\mathrm{C}-\mathrm{O}$ range, whereas the coronene-O binding region has a stable spin state has on its singlet state. We recommended that atomic $\mathrm{O}$ attack on the coronene surface started in triplet state at the "top" site with the activation energy barrier of $6.1 \mathrm{kcal} / \mathrm{mol}$, and the ISC occurs with facile oxygen atom relocation to develop a stable epoxide functional group in a singlet state of $-29.2 \mathrm{kcal} / \mathrm{mol}$ or $-35.0 \mathrm{kcal} / \mathrm{mol}$ for its interactions energy.

\section{- ACKNOWLEDGMENTS}

We would like to express our sincere gratitude to Research Directorate of Universitas Gadjah Mada who has provided research funding support through research scheme Capacity Improvement for Young Lecturer Researcher year 2019 under contract number 3943/UN1/DITLIT/DIT-LIT/LT/2019. The calculations were part performed at Austrian-Indonesian Center for Computational Chemistry and facilities of HPC LIPI, Indonesian Institute of Sciences (LIPI). This work is partially funded by the grant from Faculty of Mathematics and Natural Sciences, Universitas Gadjah Mada under contract number 87/J01.1.28/PL.06.02/ 2019 and Directorate of Research, UGM under RTA 2020 scheme. 


\section{- REFERENCES}

[1] Geim, A.K., and Novoselov, K.S., 2007, The rise of graphene, Nat. Mater., 6 (3), 183-191.

[2] Johns, J.E., and Hersam, M.C., 2013, Atomic covalent functionalization of graphene, Acc. Chem. Res., 46 (1), 77-86.

[3] Dreyer, D.R., Park, S., Bielawski, C.W., and Ruof, R.S., 2010, The chemistry of graphene oxide, Chem. Soc. Rev., 39 (1), 228-240.

[4] Wang, S., Ang, P.K., Wang, Z., Tang, A.L.L., Thong, J.T.L., and Loh, K.P., 2010, High mobility, printable, and solution-processed graphene electronics, Nano Lett., 10 (1), 92-98.

[5] Becerril, H.A., Mao, J., Liu, Z., Stoltenberg, R.M., Bao, Z., and Chen, Y., 2008, Evaluation of solutionprocessed reduced graphene oxide films as transparent conductors, ACS Nano, 2 (3), 463-470.

[6] Matyba, P., Yamaguchi, H., Eda, G., Chhowalla, M., Edman, L., and Robinson, N.D., 2010, Graphene and mobile ions: The key to all-plastic, solution-processed light-emitting devices, ACS Nano, 4 (2), 637-642.

[7] Stankovich, S., Dikin, D.A., Piner, R.D., Kohlhaas, K.A., Kleinhammes, A., Jia, Y., Wu, Y., Nguyen, S.T., and Ruoff, R.S., 2007, Synthesis of graphene-based nanosheets via chemical reduction of exfoliated graphite oxide, Carbon, 45 (7), 1558-1565.

[8] Hummers, W.S., and Offeman, R.E., 1958, Preparation of graphitic oxide, J. Am. Chem. Soc., 80 (6), 1339.

[9] Schniepp, H.C., Li, J.L., McAllister, M.J., Sai, H., Herrera-Alonso, M., Adamson, D.H., Prud'homme, R.K., Car, R., Seville, D.A., and Aksay, I.A., 2006, Functionalized single graphene sheets derived from splitting graphite oxide, J. Phys. Chem. B, 110 (17), 8535-8539.

[10] Szabó, T., Berkesi, O., Forgó, P., Josepovits, K., Sanakis, Y., Petridis, D., and Dékány, I., 2006, Evolution of surface functional groups in a series of progressively oxidized graphite oxides, Chem. Mater., 18 (11), 2740-2749.

[11] Gao, W., Alemany, L.B., Ci, L., and Ajayan, P.M., 2009, New insights into the structure and reduction of graphite oxide, Nat. Chem., 1 (5), 403-408.
[12] Li, X., Wang, H., Robinson, J.T., Sanchez, H., Diankov, G., and Dai, H., 2009, Simultaneous nitrogen doping and reduction of graphene oxide, J. Am. Chem. Soc., 131 (43), 15939-15944.

[13] Barinov, A., Malciog, O.B., Fabris, S., Gregoratti, L., Dalmiglio, M., Kiskinova, M., Baris, O., and Sun, T., 2009, Initial stages of oxidation on graphitic surfaces: Photoemission study and density functional theory calculations, J. Phys. Chem. C, 113 (21), 9009-9013.

[14] Nourbakhsh, A., Cantoro, M., Klekachev, A.V., Pourtois, G., Vosch, T., Hofkens, J., van der Veen, M.H., Heyns, M.M., De Gendt, S., and Sels, B.F., 2011, Single layer vs bilayer graphene: A comparative study of the effects of oxygen plasma treatment on their electronic and optical properties, J. Phys. Chem. C, 115 (33), 16619-16624.

[15] Nourbakhsh, A., Cantoro, M., Vosch, T., Pourtois, G., Clemente, F., van der Veen, M.H., Hofkens, J., Heyns, M.M., De Gendt, S., and Sels, B.F., 2010, Bandgap opening in oxygen plasma-treated graphene, Nanotechnology, 21 (43), 435203.

[16] Hossain, M.Z., Johns, J.E., Bevan, K.H., Karmel, H.J., Liang, Y.T., Yoshimoto, S., Mukai, K., Koitaya, T., Yoshinobu, J., Kawai, M., Lear, A.M., Kesmodel, L.L., Tait, S.L., and Hersam, M.C., 2012, Chemically homogeneous and thermally reversible oxidation of epitaxial graphene, Nat. Chem., 4 (4), 305-309.

[17] Vinogradov, N.A., Schulte, K., Ng, M.L., Mikkelsen, A., Lundgren, E., Mårtensson, N., and Preobrajenski, A.B., 2011, Impact of atomic oxygen on the structure of graphene formed on $\operatorname{Ir}(111)$ and $\operatorname{Pt}(111)$, J. Phys. Chem. C, 115 (19), 9568-9577.

[18] Srinivasan, S.G., and van Duin, A.C.T., 2011, Molecular-dynamics-based study of the collisions of hyperthermal atomic oxygen with graphene using the ReaxFF reactive force field, J. Phys. Chem. A, 115 (46), 13269-13280.

[19] Paci, J.T., Upadhyaya, H.P., Zhang, J., Schatz, G.C., and Minton, T.K., 2009, Theoretical and experimental studies of the reactions between hyperthermal $\mathrm{O}\left({ }^{3} \mathrm{P}\right)$ and graphite: Graphene-based direct dynamics and beam-surface scattering approaches, J. Phys. Chem. A, 113 (16), 4677-4685. 
[20] Morón, V., Martin-Gondre, L., Crespos, C., Larregaray, P., Gamallo, P., and Sayós, R., 2012, Classical dynamics study of atomic oxygen over graphite (0001) with new interpolated and analytical potential energy surfaces, Comput. Theor. Chem., 990, 132-143.

[21] Isborn, C.M., Li, X., and Tully, J.C., 2007, Timedependent density functional theory Ehrenfest dynamics: Collisions between atomic oxygen and graphite clusters, J. Chem. Phys., 126 (13), 134307.

[22] Steglich, M., Carpentier, Y., Jäger, C., Huisken, F., Räder, H.J., and Henning, T., 2012, The smoothness of the interstellar extinction curve in the UV: Comparison with recent laboratory measurements of PAH mixtures, Astron. Astrophys., 540, A110.

[23] Hammonds, M., Pathak, A., and Sarre, P.J., 2009, TD-DFT calculations of electronic spectra of hydrogenated protonated polycyclic aromatic hydrocarbon (PAH) molecules: Implications for the origin of the diffuse interstellar bands?, Phys. Chem. Chem. Phys., 11 (22), 4458-4464.

[24] Lebouteiller, V., Brandl, B., Bernard-Salas, J., Devost, D., and Houck, J.R., 2007, PAH strength and the interstellar radiation field around the massive young cluster NGC 3603, Astrophys. J., 665 (1), 390-401.

[25] Biennier, L., Alsayed-Ali, M., Foutel-Richard, A., Novotny, O., Carles, S., Rebrion-Rowe, C., and Rowe, B., 2006, Laboratory measurements of the recombination of $\mathrm{PAH}$ ions with electrons: Implications for the PAH charge state in interstellar clouds, Faraday Discuss., 133, 289-301.

[26] Malloci, G., Mulas, G., and Joblin, C., 2004, Electronic absorption spectra of PAHs up to vacuum UV, Astron. Astrophys., 426 (1), 105-117.

[27] Lee, M.W., and Meuwly, M., 2014, Diffusion of atomic oxygen relevant to water formation in amorphous interstellar ices, Faraday Discuss., 168, 205-222.

[28] Dulieu, F., Amiaud, L., Congiu, E., Fillion, J.H., Matar, E., Momeni, A., Pirronello, V., and Lemaire, J.L., 2010, Experimental evidence for water formation on interstellar dust grains by hydrogen and oxygen atoms, Astron. Astrophys., 512 (5), A30.
[29] Goumans, T.P.M., Catlow, C.R.A., Brown, W.A., Kästner, J., and Sherwood, P., 2009, An embedded cluster study of the formation of water on interstellar dust grains, Phys. Chem. Chem. Phys., 11 (26), 5431-5436.

[30] Tachikawa, H., 2017, Hydrogen atom addition to the surface of graphene nanoflakes: A density functional theory study, Appl. Surf. Sci., 396, 1335-1342.

[31] Tachikawa, H., and Kawabata, H., 2019, Additions of fluorine atoms to the surfaces of graphene nanoflakes: A density functional theory study, Solid State Sci., 97, 106007.

[32] Tachikawa, H., 2020, Mechanism of Li storage on graphene nanoflakes: Density functional theory study, Surf. Sci., 691, 121489.

[33] Hutama, A.S., Hijikata, Y., and Irle, S., 2017, Coupled cluster and density functional studies of atomic fluorine chemisorption on coronene as model systems for graphene fluorination, J. Phys. Chem. C, 121 (27), 14888-14898.

[34] Wang, Y., Qian, H.J., Morokuma, K., and Irle, S., 2012, Coupled cluster and density functional theory calculations of atomic hydrogen chemisorption on pyrene and coronene as model systems for graphene hydrogenation, J. Phys. Chem. A, 116 (26), 7154-7160.

[35] Lee, C., Yang, W., and Parr, R.G., 1988, Development of the Colle-Salvetti correlation-energy formula into a functional of the electron density, Phys. Rev. B: Condens. Matter, 37 (2), 785-789.

[36] Frisch, M.J., Trucks, G.W., Schlegel, H.B., Scuseria, G.E., Robb, M.A., Cheeseman, J.R., Scalmani, G., Barone, V., Mennucci, B., Petersson, G.A., Nakatsuji, H., Caricato, M., Li, X., Hratchian, H.P., Izmaylov, A.F., Bloino, J., Zheng, G., Sonnenberg, J.L., Hada, M., Ehara, M., Toyota, K., Fukuda, R., Hasegawa, J., Ishida, M., Nakajima, T., Honda, Y., Kitao, O., Nakai, H., Vreven, T., Montgomery Jr., J.A., Peralta, J.E., Ogliaro, F., Bearpark, M., Heyd, J.J., Brothers, E., Kudin, K.N., Staroverov, V.N., Kobayashi, R., Normand, J., Raghavachari, K., Rendell, A., Burant, J.C., Iyengar, S.S., Tomasi, J., Cossi, M., Rega, N., Millam, J.M., Klene, M., Knox, J.E., Cross, J.B., 
Bakken, V., Adamo, C., Jaramillo, J., Gomperts, R., Stratmann, R.E., Yazyev, O., Austin, A.J., Cammi, R., Pomelli, C., Ochterski, J.W., Martin, R.L., Morokuma, K., Zakrzewski, V.G., Voth, G.A., Salvador, P., Dannenberg, J.J., Dapprich, S., Daniels, A.D., Farkas, Ö., Foresman, J.B., Ortiz, J.V., Cioslowski, J., and Fox, D.J., 2013, Gaussian-09 Revision D.01, Gaussian, Inc., Wallingford, CT.

[37] Manz, T.A., 2017, Introducing DDEC6 atomic population analysis: Part 3. Comprehensive method to compute bond orders, RSC Adv., 7 (72), 45552-45581.

[38] Manz, T.A., and Limas, N.G., 2016, Introducing DDEC6 atomic population analysis: Part 1. Charge partitioning theory and methodology, RSC Adv., 6 (53), 47771-47801.

[39] Limas, N.G., and Manz, T.A., 2016, Introducing DDEC6 atomic population analysis: Part 2. Computed results for a wide range of periodic and nonperiodic materials, RSC Adv., 6 (51), 4572745747.

[40] Tachikawa, H., Iyama, T., and Kawabata, H., 2013, Interaction of hydroxyl $\mathrm{OH}$ radical with graphene surface: A density functional theory study, Jpn. J. Appl. Phys., 52 (1S), 01AH01.

[41] Tachikawa, H., and Kawabata, H., 2011, Ground and low-lying excited electronic states of graphene flakes: a density functional theory study, J. Phys. B: At. Mol. Opt. Phys., 44 (20), 205105.

[42] Li, J.L., Kudin, K.N., McAllister, M.J., Prud'homme, R.K., Aksay, I.A., and Car, R., 2006, Oxygen-driven unzipping of graphitic materials, Phys. Rev. Lett., 96 (17), 176101.

[43] Blöchl, P.E., 1994, Projector augmented-wave method, Phys. Rev. B, 50 (24), 17953-17979.

[44] Perdew, J.P., Burke, K., and Ernzerhof, M., 1996, Generalized gradient approximation made simple, Phys. Rev. Lett., 77 (18), 3865-3868.

[45] Watanabe, K., Nakatani, N., Nakayama, A., Higashi, M., and Hasegawa, J., 2016, Spin-blocking effect in $\mathrm{CO}$ and $\mathrm{H}_{2}$ binding reactions to molybdenocene and tungstenocene: A theoretical study on the reaction mechanism via the minimum energy intersystem crossing point, Inorg. Chem., 55 (16), 8082-8090.

[46] Kitagawa, Y., Chen, Y., Nakatani, N., Nakayama, A., and Hasegawa, J., 2016, A DFT and multiconfigurational perturbation theory study on $\mathrm{O}_{2}$ binding to a model heme compound via the spinchange barrier, Phys. Chem. Chem. Phys., 18 (27), 18137-18144.

[47] Ma, Z., Ukaji, K., Nakatani, N., Fujii, H., and Hada, M., 2019, Substitution effects on olefin epoxidation catalyzed by Oxoiron(IV) porphyrin $\pi$-cation radical complexes: A DFT study, J. Comput. Chem., 40 (19), 1780-1788.

[48] Ma, Z., Nakatani, N., Fujii, H., and Hada, M., 2020, Effect of external electric fields on the oxidation reaction of olefins by $\mathrm{Fe}(\mathrm{IV}) \mathrm{OCl}$-porphyrin complexes, Bull. Chem. Soc. Jpn., 93 (2), 187-193.

[49] Wang, Y., Qian, H., Wu, Z., and Irle, S., 2017, $\mathrm{QM} / \mathrm{MD}$ simulations on graphene hydrogenation/deuteration: $\mathrm{C}_{x} \mathrm{H} / \mathrm{D}$ formation mechanism and isotope effect, J. Phys. Chem. C, 121 (15), 8480-8489.

[50] Fu, B., Han, Y., Bowman, J.M., Leonori, F., and Balucani, N., 2012, Experimental and theoretical studies of the $\mathrm{O}\left({ }^{3} \mathrm{P}\right)+\mathrm{C}_{2} \mathrm{H}_{4}$ reaction dynamics: Collision energy dependence of branching ratios and extent of intersystem crossing, J. Chem. Phys., 137 (22), 22A532.

[51] Fu, B., Han, Y.C., Bowman, J.M., Angelucci, L., Balucani, N., Leonori, F., and Casavecchia, P., 2012, Intersystem crossing and dynamics in $\mathrm{O}\left({ }^{3} \mathrm{P}\right)+\mathrm{C}_{2} \mathrm{H}_{4}$ multichannel reaction: Experiment validates theory, Proc. Natl. Acad. Sci. U.S.A., 109 (25), 9733-9738.

[52] Hu, W., Lendvay, G., Maiti, B., and Schatz, G.C., 2008, Trajectory surface hopping study of the $\mathrm{O}\left({ }^{3} \mathrm{P}\right)$ + ethylene reaction dynamics, J. Phys. Chem. A, 112 (10), 2093-2103.

[53] Reed, A.E., Curtiss, L.A., and Weinhold, F., 1988, Intermolecular interactions from a natural bond orbital, donor-acceptor viewpoint, Chem. Rev., 88 (6), 899-926. 